\title{
Detection of consensus genomic regions associated with root architecture of bread wheat on groups 2 and 3 chromosomes using QTL meta-analysis
}

\author{
Hadi Darzi-Ramandi ${ }^{1,2}$, Vahid Shariati $\mathrm{J}^{*^{2}}$, Elahe Tavakol ${ }^{* 3}$, Hamid Najafi-Zarini ${ }^{1}$, Sayedeh Saba \\ Bilgrami $^{2,4}$, Khadijeh Razavi ${ }^{2}$
}

${ }^{1}$ Department of Plant Breeding and Biotechnology, Sari University of Agricultural Sciences and Natural Resources (SANRU), Sari, Iran

${ }^{2}$ Department of Plant Molecular Biotechnology, National Institute of Genetic Engineering and Biotechnology (NIGEB), Tehran, Iran

${ }^{3}$ Department of Crop Production and Plant Breeding, Shiraz University, Shiraz, Iran

${ }^{4}$ Department of Plant Breeding and Biotechnology, University of Zabol, Zabol, Iran

\section{*Corresponding authors: elahetavakol@gmail.com; vshariati@nigeb.ac.ir}

\begin{abstract}
Root architecture is an important bread wheat phenomenon that highly influences its production and adaptation to environmental stresses, in particular drought stress. Several QTL studies have been conducted to ascertain chromosomal regions associated with root morphology resulting in identification of various loci depending on evaluated population types and experimental conditions. In order to identify the most consistent and reliable QTLs involved in various root morphological traits in bread wheat, a meta-QTL (MQTL) analysis was performed using 106 QTLs derived from 12 different populations under both normal and drought stress conditions. Among them, 125 QTLs related to root traits were successfully projected onto the reference map and further metaanalysis was focused on chromosomes of homeologous groups 2 and 3 with most assigned QTLs. Consequently, a total of seven MQTLs were identified on chromosomes 2A, 2B, 3A and 3B originated from 2 to 17 initial QTLs with a confidence interval (CI) of 5.3-6.6 to 39.5-55.0 cM. Three MQTLs located on 2A, 3A and 3B derived from 7 to 17 QTLs related to different root morphological traits pointed out the most important chromosomal regions. A reduction in the average $95 \%$ confidence interval from $20.8 \mathrm{cM}$ to 6.4 $\mathrm{cM}$ was observed when comparing the individual QTL to the MQTL. Further analysis on investigation of candidate genes located in these genomic regions resulted in identification of some genes mainly associated with lignin catabolic process, potassium transporters and leucine-rich repeats receptor-like kinases (LRR-RLKs). These results provid fundamental information on most important genomic regions and candidate genes related to root morphology in bread wheat.
\end{abstract}

Keywords: Bread wheat; Root traits; Quantitative trait loci; Meta-QTL analysis.

Abbreviations: QTL_quantitative trait loci; MQTL_meta-QTL; CI_confidence interval; ${ }^{2}$ _phenotypic variance explained by the QTL; LOD_logarithm of odds; AIC_akaike information criterion; RL_root length; RSA_root surface area; RV_root volume; RN_root number; RDW_root dry weight; BC_backcrossed lines; DH_doubled haploid lines; RIL_recombinant inbred lines.

\section{Introduction}

Root morphological traits are fundamental for multiple functions that are at the basis of plant productivity, which including efficiency of water and inorganic nutrients absorption, root development directly affects the growth and biomass production, and is important factor of the high and stable yield in plants (Sharma et al., 2011). The effect of root architecture on yield and related traits and its role in increasing drought tolerance have been widely reported for all major crops (Tuberosa et al., 2002a, b; de Dorlodot et al., 2007, Christopher et al., 2013). Growth and development of root are not only affected by climate and cultivations condition but also controlled by numerous genes. Caradus (1995) suggested that the traits associated with root size such as root length, weight, volume, number, surface area, and the ratio of root to shoot dry weight have the highest heritability in compare to other root morphological traits such as root diameter, density, length of hairs. Several studies revealing the extent and nature of root morphological genetic variation have profound implications for improving water- and nutrient-use efficiency of crops or for enhancing their productivity under abiotic stresses or suboptimal soil conditions.

Genotypes with the greatest root vigor under drought conditions showed the lowest yield reductions under severe water stress (Motzo et al., 2013). The previous studies indicated that root architecture determined yield potential under drought conditions; for example, high yielding upland rice varieties with longer root length. (Steele et al. 2007). Although root traits have vital effects on plant yield under water-limited conditions, they are rarely considered as selection parameter for improvement of wheat and other plants because they are difficult to measure. In general it can be said that the inherent difficulties in root system evaluation (because of the quantitative nature of root characteristics, their complex genetic control, and the strong environmental effects) has so far slowed the pace of mutants, genes and 
QTL discovery for roots and lead to a paucity of root traitsassociated molecular markers to be used in MAS. This has largely precluded breeding programs to exploit root traits in breeding. The genetic variation observed for root morphology characters has been used to study the inheritance of various root traits under control and drought stress conditions (Richard et al., 2105; Chapagain et al., 2014; Comas et al., 2013; Whalley et al., 2013). However, only limited information available for the genetic control of root characters in wheat. As per the Monyo and Whttington (1970) reports the single genes, as well as polygenic systems controlling growth period, had an effect on both root and shoot. MacKey (1973) demonstrated that genetic control of root traits was spread over the whole genome by using a set of monosomics lines in bread wheat. Richards and Passioura (1981) observed that control of 3 traits i.e., maximum xylem vessel diameter, number of seminal root axes, and number of metaxylem vessels in bread wheat was multigenetic. Ehdai and Waines (1997) had used a set of ditelosomic and dimonotelosomic lines of Chinese Spring, and demonstrated that the expression of root biomass in bread wheat had been affected by at least 13 chromosome arms belonging to the A, $\mathrm{B}$, and $\mathrm{D}$ genomes.

Genomic loci controlling such traits are called quantitative trait loci (QTL). With the advent and development of molecular markers, this capability is established that estimate the gene position and size of QTL, including those for root morphology traits (Sharma et al. 2011). The applications of molecular marker techniques and outcomes of quantitative trait locus (QTL) mapping have facilitated a better understanding of the genetic basis of root traits and grain yield. Identification of QTLs associated with wheat root morphology provides useful information for avoiding drought stress and maintaining yield production under the irrigation condition. In some cases, these studies have suggested possible roles for these QTL in determining plant yield, due to the overlap of QTL for root features with those for traits related to productivity (Tuberosa et al., 2002a, b; Steele et al., 2007). A number of QTLs for root traits in rice (Horii et al. 2005; Steele et al., 2007), barley (Arifuzzaman et al., 2014), maize (Tuberosa et al., 2002b; Burton et al., 2015), durum wheat (Maccaferri et al., 2016) have been mapped in many studies. The more and fresh amount of data for specific QTL, enhances our physiological and evolutionary understanding and reveals links between root morphology and root functions that will be essential in designing root surface aria for target environments. For instance, a major QTL for root length of chromosome 9 in rice, which has been deployed in smart breeding and marker-assisted selection (Steele et al., 2006), has shown a degree of QTL by environment interaction under drought that suggests its greater effectiveness in limited water conditions. The same QTL is also associated with the size and volume of individual adventitious root axes, rather than branching of seminal roots (Horii et al., 2005). Overlap of QTLs for root system morphology in maize with those for grain yield suggests the possible role of these QTL in determining the grain yield (Tuberosa et al., 2002b).

Since different populations (Austin et al., 2000), generations ( $\mathrm{Li}$ et al., 2007), and environmental conditions (Li et al., 2003; Lan et al., 2005) were commonly found to have great influence on the results of QTL detection experiments, direct comparisons of QTLs data across different studies were difficult. The integration of QTL data from diverse resources is informative for the eventual QTL cloning and breeding application. Meta-analysis is a method of combining data from different sources in a single study to determine co-locations between genes and QTLs (Goffinet and Gerber 2000; Arcade et al., 2004). Pooling of results from several studies allows greater statistical power for QTL detection and more precise estimation of their genetic effects. Hence, a meta-analysis can yield conclusions that are stronger than those of individual studies and can give greater insight into the genetic architecture of complex traits $(\mathrm{Wu}$ and $\mathrm{Hu}, 2012$ ). This approach has been used to study different quantitative traits in various cereals such as abiotic stresses tolerance in barley (Zhang et al., 2016), yield in rice (Wu et al., 2016), and disease resistance in maize (Zhao et al., 2015). Meta-analysis of QTLs has been also employed for root architecture in rice (Courtois et al., 2009), Brassica napus (Zhang et al., 2016) and adaptation to drought and heat stress in bread wheat (Acuña-Galindo et al., 2014). Despite of different studies, research is limited in using meta-analyses for studying root system morphology in wheat. Therefore, the objectives of this study were to identify, using meta-QTL analysis, chromosomal regions where 'real' QTL involved in traits related to root morphology are located that will aid breeders in targeted genetic improvement .

\section{Results}

Distribution of initial QTLs associated with traits of root morphology

The QTLs used in this study derived from various population types included backcrossed lines (BC), recombinant inbred lines (RIL), doubled haploid lines (DH) and $\mathrm{F}_{2}$ plants and the population sizes ranged from 85 to 206 plants (Table 1). A total of 243 QTLs related to root morphology were identified in the 12 experiments subjected to the current meta-analysis. The number of QTLs on each chromosome varied between 1 (chromosome 5B, 6B and 6D) to 31 (chromosome 1A) (Supplementary Table 1). Among them, 125 QTLs with sufficient information and common markers with the reference map including 105 QTLs in normal conditions and 20 QTLs under drought stress conditions were selected for further analysis (Fig 1).

Of the 105 QTLs in normal conditions 61 QTLs of 10 populations were associated with root length and root number, 28 QTLs of six populations with root surface area, root volume and 16 QTLs of five populations with root dry weight, roots angle and root diameter (Fig 1). Of the seven traits associated with root morphology, root length (13.4\%) and roots angle $(2.1 \%)$ had the highest and the lowest average $\mathrm{R}^{2}$ value, respectively (Fig 2). LOD scores were ranged from 2.05 to 29.9, for QTLs associated with root volume and root length, respectively. Average LOD for all traits was estimated as 4.6 (Table 2). Most of the QTLs for root traits were located on homoeologous group 2 and 3 chromosomes with 35 and 18, respectively (Table 3). QTLs for root length (12 QTLs) and root surface area (7 QTLs) were mainly on homoeologous group 2 and 3 chromosomes and QTLs for root number (10 QTLs) and root volume (7 QTLs) were mainly located on homoeologous groups 2 and 3 chromosomes. Due to presence of most root related QTLs on homoeologous group 2 and 3 chromosomes, they were subjected to further analysis of metaQTL.

\section{Meta-analysis of QTLs for root morphology}

A total of 53 QTLs related to root morphology on homoeologous groups 2 and 3 chromosomes were anchored 
Table 1. The origin of QTLs related to root morphological traits included in the meta-analysis.

\begin{tabular}{|c|c|c|c|c|c|c|c|}
\hline Parents of Population & $\begin{array}{l}\text { Population } \\
\text { size }\end{array}$ & Population type & $\begin{array}{l}\text { Number of } \\
\text { marker }\end{array}$ & $\begin{array}{l}\text { Map } \\
\text { density }\end{array}$ & Marker type & $\begin{array}{l}\text { Experiment } \\
\text { environments }\end{array}$ & Reference \\
\hline Weimai $8 \times$ Luohan 2 & 179 & RILs & 496 & 2.64 & SSR & $\mathrm{N}$ and DS & Zhang et al., 2014 \\
\hline Weimai $8 \times$ Yannong 19 & 175 & RILs & 496 & 2.64 & SSR & $\mathrm{N}$ and DS & Zhang et al., 2014 \\
\hline Weimai $8 \times$ Jimai 20 & 172 & RILs & 496 & 2.64 & SSR & $\mathrm{N}$ and DS & Zhang et al., 2014 \\
\hline Chinese Spring $\times$ SQ1 & 90 & DH & 338 & 8.45 & SSR & $\mathrm{N}$ and DS & Czyczyło-Mysza et al., 2013 \\
\hline Xiaoyan54 $\times$ Jing 411 & 142 & RILs & 470 & 7.3 & SSR & $\mathrm{N}$ & Ren et al., 2012 \\
\hline Creso $\times$ Pedroso & 123 & $\mathrm{~F}_{7}$ & 600 & 3 & SSR \& DArT & $\mathrm{N}$ & Petraruloet al., 2014 \\
\hline WL711 $\times$ C306 & 206 & $\operatorname{RIL}\left(\mathrm{F}_{9} / \mathrm{F}_{10}\right)$ & 173 & 21.2 & SSR \& STS & N \& DS & Kadam et al., 2012 \\
\hline Mironovskaya808 $\times$ Chinese Spring & 100 & $\mathrm{~F}_{2}$ & 122 & 19.5 & SSR \& SNP & $\mathrm{N}$ and $\mathrm{ABA}$ treatment & Iehisaet al., 2014 \\
\hline Hanxuan $10 \times$ Lumai 14 & 150 & DH & 395 & 9.9 & SSR \& RFLP & $\mathrm{N}$ and DS & Liu et al., 2013 \\
\hline Opata85 $\times$ W7984 & 114 & RILs & 800 & 4.4 & SSR & $\mathrm{N}$ and $\mathrm{PEG}^{\mathrm{b}}$ & Landjeva et al., 2013 \\
\hline Chinese Spring $\times$ Synthetic 6 & 85 & $\mathrm{DILs}^{\mathrm{a}}$ & 80 & 6.87 & SSR & $\mathrm{N}$ and $\mathrm{PEG}$ & Landjeva et al., 2008 \\
\hline Huapei3 × Yumai57 & 168 & DH & 323 & 7.67 & SSR \& EST & $\mathrm{N}$ & Zhao et al., 2014 \\
\hline
\end{tabular}

Huapei3 $\times$ Yumai57

yethylene glycol

$\square$ Drought stress $\square$ Normal

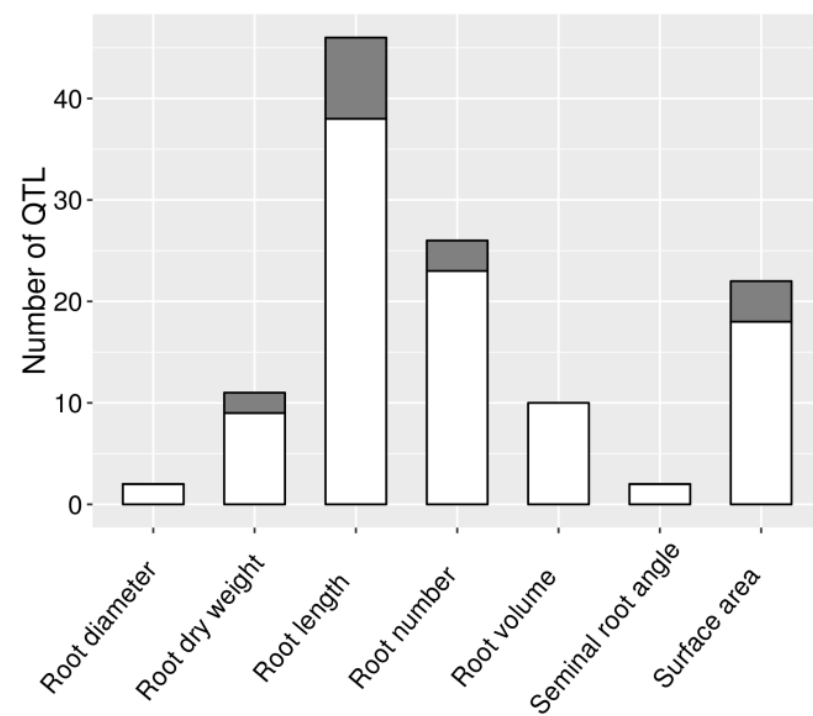

Fig 1. Number of QTLs associated with root morphological traits under normal and drought stress conditions in 12 different populations. 
Table 2. Number of QTLs related to root morphology identified on each chromosome.

\begin{tabular}{lcccccccc}
\hline \multirow{2}{*}{ Wheat genome } & \multicolumn{7}{c}{ Chromosome number } & \multirow{2}{*}{ Total } \\
\cline { 2 - 8 } & 1 & 2 & 3 & 4 & 5 & 6 & 7 & \\
\hline $\mathrm{A}$ & $30 / 13$ & $19 / 17$ & $14 / 9$ & $5 / 4$ & $4 / 2$ & $15 / 8$ & $6 / 2$ & $55 / 93$ \\
$\mathrm{~B}$ & $16 / 2$ & $23 / 14$ & $25 / 6$ & $11 / 7$ & $14 / 6$ & $11 / 6$ & $11 / 4$ & $45 / 111$ \\
$\mathrm{D}$ & $1 / 1$ & $4 / 4$ & $4 / 4$ & $4 / 4$ & $9 / 4$ & $8 / 4$ & $11 / 4$ & $25 / 41$ \\
Total & $47 / 16$ & $46 / 35$ & $43 / 19$ & $20 / 15$ & $27 / 12$ & $34 / 18$ & $28 / 10$ & $125 / 245$ \\
\hline
\end{tabular}

Numbers after and before slash indicate number of QTLs identified in evaluated studies and used in meta-QTL analysis, respectively.

Table 3. List of QTLs associated with root morphological traits on 2A, 2B, 3A and 3B chromosomes used for the QTL metaanalysis.

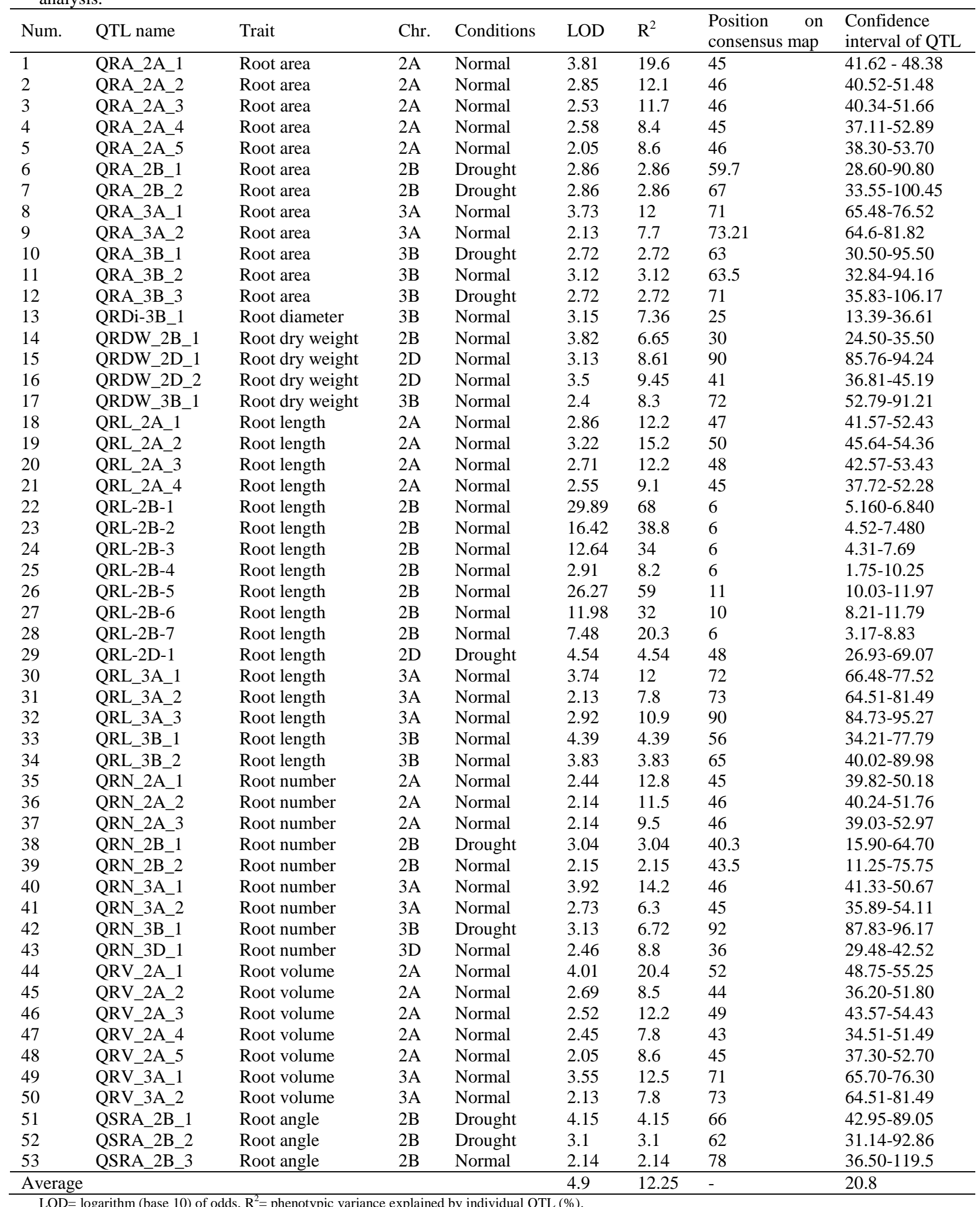




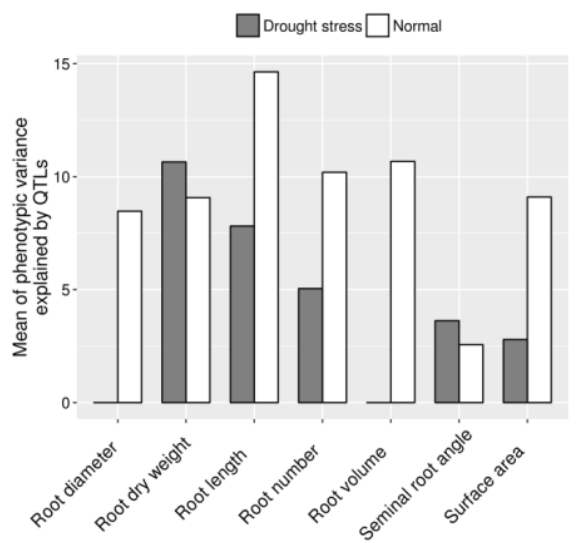

Fig 2. The average variance explained by the QTLs $\left(\mathrm{R}^{2}\right)$ associated with root morphological traits under normal and drought stress conditions.
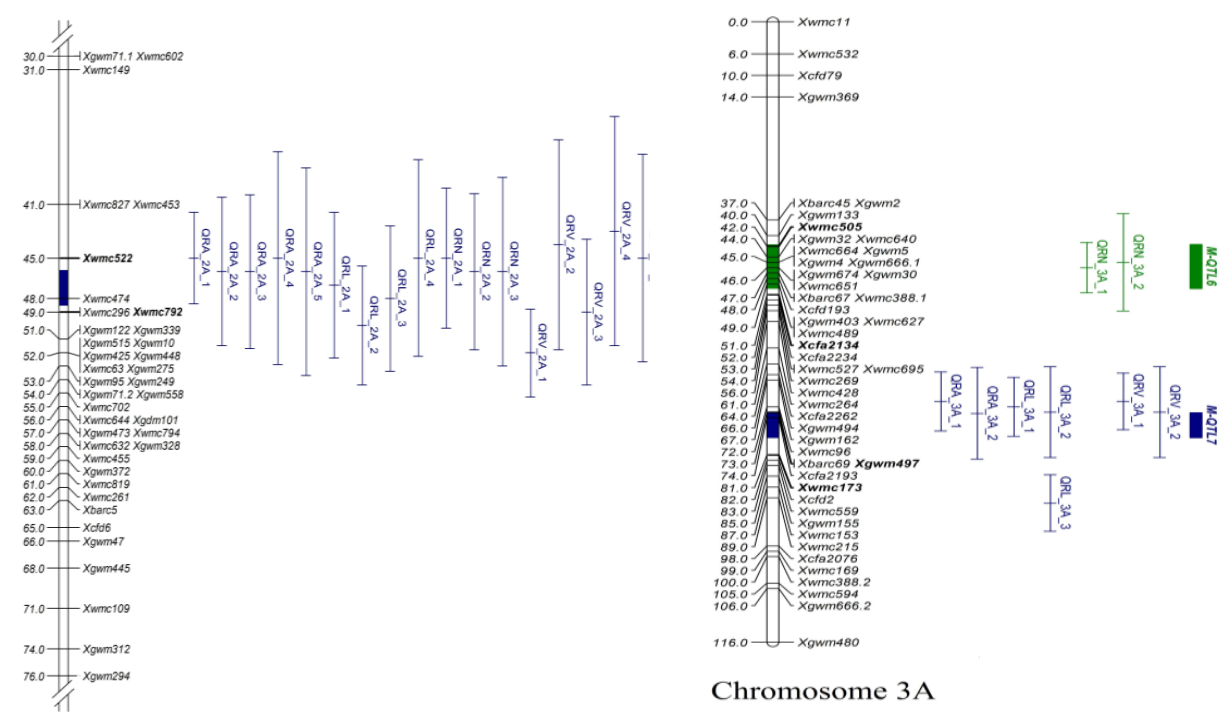

Chromosome $3 \mathrm{~A}$

Chromosome 2A

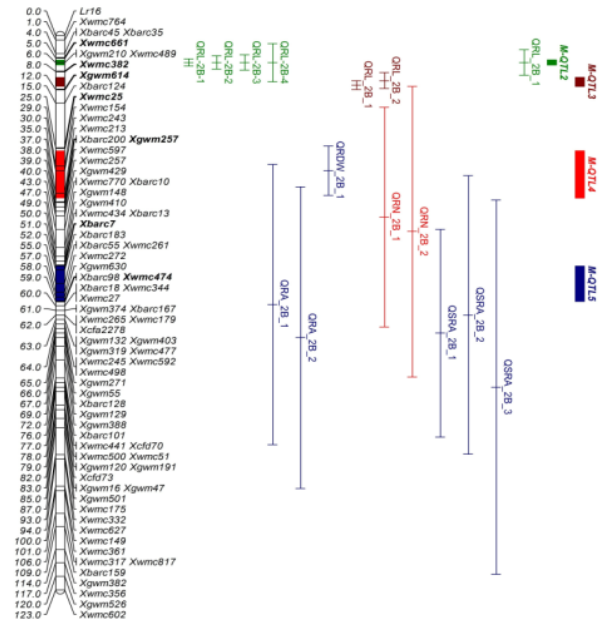

Chromosome 2B

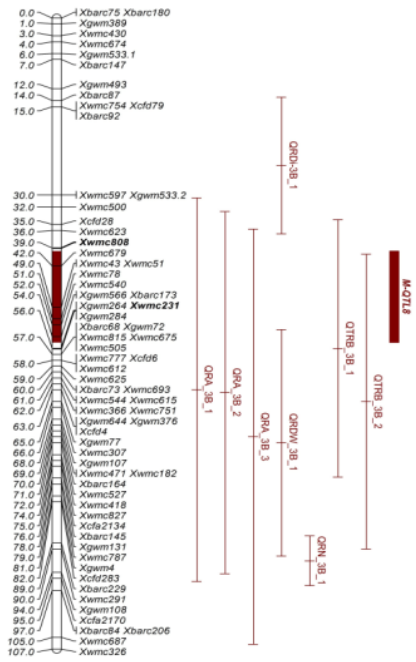

Chromosome 3B

Fig. 3. Chromosomal locations of QTLs with $95 \%$ confidence intervals associated with root morphological traits on chromosomes 2A, 2B, 3A and 3B in bread wheat. Markers name are on the right; the distances in centiMorgan are on the left. Chromosomes were referenced from the wheat consensus map of Somers et al. (2004). The BioMercator program used to integration genetic maps and QTLs towards discovery of MQTLs. 
Table 4. Consensus QTLs of six root morphological traits identified by meta-analysis on 2A, 2B, 3A and 3B chromosomes.

\begin{tabular}{|c|c|c|c|c|c|c|c|}
\hline Chr. & MQTL & $\underset{\mathrm{a}}{\mathrm{AIC}}$ & $\begin{array}{l}\text { Position on } \\
\text { consensus } \\
\text { map }(\mathrm{cM})\end{array}$ & $\begin{array}{c}\text { Confidence } \\
\text { interval M-QTL }\end{array}$ & $\begin{array}{c}\text { Flanking markers of } \\
\text { the position }\end{array}$ & $\begin{array}{l}\text { Number of } \\
\text { initial QTLs }\end{array}$ & Trait \\
\hline $2 \mathrm{~A}$ & M-QTL1 & 86.5 & 47.2 & $45.9-48.5$ & Xwmc522-Xwmc792 & 17 & $\begin{array}{l}\text { RV, RSA, } \\
\text { RL, RN }\end{array}$ \\
\hline \multirow{4}{*}{$2 \mathrm{~B}$} & M-QTL2 & $\begin{array}{c}112 . \\
1\end{array}$ & 6.0 & $5.3-6.6$ & Xwmc661-Xwmc382 & 5 & RL \\
\hline & M-QTL3 & $\begin{array}{c}112 . \\
1\end{array}$ & 10.8 & $9.9-11.6$ & Xwmc382-Xgwm614 & 2 & RL \\
\hline & M-QTL4 & $\begin{array}{c}112 . \\
1\end{array}$ & 30.8 & $25.5-36.1$ & $X w m c 25-X g w m 257$ & 3 & $\mathrm{RN}$ \\
\hline & M-QTL5 & $\begin{array}{c}112 . \\
1\end{array}$ & 59.0 & $51.9-59.0$ & Xbarc7-Xwmc474 & 5 & RSA \\
\hline \multirow{2}{*}{$3 \mathrm{~A}$} & M-QTL6 & 58.7 & 45.8 & $41.6-49.9$ & Xwmc505-Xcfa2134 & 2 & $\mathrm{RN}$ \\
\hline & M-QTL7 & 58.7 & 75.5 & $73.1-77.8$ & Xgwm497-Xwmc173 & 8 & RV, RSA, RL \\
\hline $3 \mathrm{~B}$ & M-QTL8 & 86.8 & 47.3 & $39.5-55.0$ & Xwmc808-Xwmc231 & 7 & $\begin{array}{l}\text { RV, RSA, } \\
\text { RL,RDW }\end{array}$ \\
\hline
\end{tabular}

a. The Akaike information criterion is a criterion for model selection used to regularized maximum likelihood (ML) estimators.

onto the consensus genetic map (Somer et al., 2004) and utilized in meta-QTL analysis. Over the past decade, the hexaploid wheat microsatellite-based consensus linkage map by Somers et al. (2004) has been widely used as a reference for bread wheat genomics studies. As a result eight significant MQTLs were identified according to the lowest Akaike information criterion (AIC) values (Fig 3). Isolated MQTLs were distributed unevenly on group 2 and 3 wheat homoeologous chromosomes: one MQTL on chromosome $2 \mathrm{~A}$; four on chromosome $2 \mathrm{~B}$, and two MQTLs on chromosomes $3 \mathrm{~A}$ and one MQTL on chromosome 3B named as MQTL1 to MQTL8 according to their chromosomal locations (Table 4; Fig. 3). Of these identified MQTLs, five were derived from at least five initial QTLs, five with confidence interval no more than $5 \mathrm{cM}$, and seven with $\mathrm{R}^{2}$ value of more than $20.0 \%$. Among them MQTL1, MQTL2, MQTL3 and MQTL7 derived from more than five initial QTLs with confidence interval (CIs) of less than $5 \mathrm{cM}$ on chromosome 2A, 2B, 2B and 3A, respectively. MQTL1 located on the long arm of chromosome $2 \mathrm{~A}$ was detected for various root morphological traits including root number, root length, root surface area and root volume that explains $11.8 \%$ of phenotypic variation with a confidence interval of 2.61 cM. This MQTL was consistently detected in the same marker interval Xwmc522-Xwmc792 (bin 45.93-48.54) at Meta-QTL analysis. Moreover, four MQTLs (MQTL2, MQTL3, MQTL4 and MQTL5) were identified by an integrated meta-analysis of 7 root length, 3 root number, and 5 root surface area QTLs. MQTL2 and MQTL3 were associated with root length. MQTL2 on short arm of chromosome 2B merged five QTLs from four populations, with flanking markers Xwmc661-Xwmc382 at $5.3 \mathrm{cM}$ and 6.6 $\mathrm{cM}$ of the consensus map, a CI of $1.3 \mathrm{cM}$ and a $\mathrm{R}^{2}$ value of $15.8 \%$. MQTL3 on short arm of chromosome 2B merged two QTLs, predicted from five populations, into a single consensus QTL. The flanking markers for this MQTL lay at $9.9 \mathrm{cM}$ and $11.6 \mathrm{cM}$ (Xwmc382-Xgwm614), the CI of this MQTL was $1.7 \mathrm{cM}$, and its $\mathrm{R}^{2}$ value was estimated as $9.4 \%$. MQTL4 on short arm of chromosome 2B merged three QTLs, predicted from two populations, into a single consensus QTL. The flanking markers (Xwmc25-Xgwm257) for this site lay at 25.5 and $36.1 \mathrm{cM}$, the CI of the QTL was $4.6 \mathrm{cM}$, and its $\mathrm{R}^{2}$ value was $8.7 \%$. MQTL5 on short arm of chromosome 2B combined five QTLs, predicted from three populations, into a single consensus QTL. The flanking markers for this site lay at 51.9 and $59.0 \mathrm{cM}$, the CI of the QTL was $7.1 \mathrm{cM}$, and its $\mathrm{R}^{2}$ value was $14.8 \%$.

Meta-analysis was carried out on root morphology traits on chromosome 3A to explaining the number of meta-QTLs for the trait and gets an accurate estimate of their position. A total of 10 individual QTLs on chromosome 3A were used to explore MQTLs and their accurate position of root morphological traits. As results, two MQTLs (MQL6 and MQL7) were identified within an interval of $36 \mathrm{cM}$ (Table 4; Fig. 3), at $45.8 \mathrm{cM}$ (bin Xwmc505-Xcfa2134) and $75.5 \mathrm{cM}$ (bin Xgwm497-Xwmc173) distance according to the reference consensus map. MQTL6 was associated with number of roots. MQTL6 and MQTL7 comprised two and eight initial QTLs, each with a large CI, resulting in broad MQTL CI $>25 \mathrm{cM}$, respectively. On chromosome 3B, eight QTLs were projected (Table 4; Fig 3), resulted in identification of MQTL8 comprising six and two initial QTLs with positions at 47.3 and $109.1 \mathrm{cM}$, respectively. They were associated with root length, root surface area, root volume and root dry weight (Fig. 3). The marker interval for this MQTL lay at $9.9 \mathrm{cM}$ and $11.6 \mathrm{cM}$ (between SSR markers Xwmc 808 and Xwmc231), the CI of this QTL was $1.7 \mathrm{cM}$.

\section{Discussion}

In the near future, cereal crop production will face two major challenges, (i) the sharp increase in cereal production required by the growing world population, and (ii) the ongoing climate change and global warming that result in increased extreme drought and heat episodes. In this scenario, enhancing the genetic capacity of the plant to acquire soil resources (water and nutrients) is a primary target and can be accomplished by including the crop root system in the list of traits of interest for plant breeders (Lobet et al., 2013). From a methodological standpoint, phenotyping roots of crops is highly cost effective for evaluating hundreds of genotypes as required in QTL discovery studies (Maccaferri et al., 2016). The role of root architecture in plant performance in the field is indicated by the coincidence of root QTL and other agronomic importance traits. A meta-analysis combines results from multiple QTL studies, allows confirmation of QTL locations across genetic backgrounds and environments and providing more insight into genomic region associated with traits. To date, there are few reports about QTL metaanalysis for different traits in wheat (Mao et al., 2010), rice (Khowaja et al., 2009), barley (Li et al., 2013), soybean (Qi 
et al., 2011) and maize (Wang et al., 2015; Hao et al., 2010). In this study, we performed meta-QTL analysis on root morphological traits as an important part of wheat architecture with high impact on the plant yield and adaptation, but less studied due to some practical difficulties. Finding MQTL that explains several root traits at the sometime can be useful to combine, or to pyramid, a number of root characteristics by marker assisted selection (MAS).

Consequently, we combined the results of 53 root QTLs studies under both normal and drought stress conditions in wheat that resulted in identification of eight MQTLs on groups 2 and 3 chromosomes. The total length of wheat linkage map used in this study was $1086.9 \mathrm{cM}$. Nine QTLs with $\mathrm{R}^{2}$ value higher than $15 \%$ were integrated in to these MQTLs. The confidence interval at all MQTLs ranged from 1.4 to $15.5 \mathrm{cM}$ (Table 4). Three meta-QTLs, MQTL1, MQTL2 and MQTL7, were much more important. Each of these included 7-17 initial QTLs reflecting clusters of multiple QTLs for 3-4 root morphological traits.

The integrated map used to conduct the MQTL analysis is prosperous in molecular markers that correspond to gene sequences, and that can give indications on feasible candidate genes for more studies (Marone et al., 2013). Markerassisted selection (MAS) is an approach of selecting desirable individuals in a breeding program based on DNA molecular markers patterns instead of, or in addition to, their traits values. The functional markers in wheat have been predominantly generated from individual QTL studies. The candidate genes, especially those co-located with the ranked meta-QTL could be strong candidates for developing suitable markers for root morphological trait (Li et al. 2013). Information was recovered for the putative functions of sequences relevant to the molecular markers underneath the individual QTL and MQTL identified for these root traits. Further analysis revealed that under MQTL1 on chromosome 2A, SSR marker Xwmc522 is broadly identical to a member of potassium transporter gene family in Arabidopsis. In plants, individual genes are involved in soil potassium availability and uptake and possibly auxin distribution in roots (Gierth and Mäser, 2007). The characterization of Arabidopsis mutants defective in AtKUP/HAK/KT transporter disclosed that these genes affect developmental processes mainly involved in cell elongation and development of roothairs. Rigas et al. (2001) and Vicente-Agullo et al. (2004) also reported prevention of Tiny Root Hair 1 (TRH1) leads root development and stops root-hair elongation due to damaged auxin transport agravitropic. The microsatellite marker Xwmc661, under MQTL2 on chromosome 2B, corresponds to a putative LRR receptor-like serine/threonineprotein kinase. Receptor-like kinases including leucine-rich repeats (LRR-RLKs) form a large part of the RLK family in the plant genome, and comprise of three domains: an extracellular LRR domain, a single transmembrane domain, and a cytoplasmic serine/threonine kinase domain (Shiu and Bleecker, 2001). It is shown that LRR-RLKs have a key role in diverse signal transduction pathways involved in plant growth and development. Three CLAVATA1-related receptor kinases, BARELY ANY MERISTEM 1 (BAM1), $B A M 2$ and $B A M 3$, act in a positive way in the development of shoot and root apical meristems (DeYoung et al., 2006). HYPERNODULATIONABERRANT ROOT FORMATIONI $(H A R l)$ is another gene from this family that is taken part in root development and nodulation process. The HARl locus in Lotus japonicus plants changes the phenotype of roots and a hyper-nodulation. Root structure alteration is due to inhibition in root length elongation, radial expansion and increase in lateral and seminal root initiation (Wopereis et al., 2000).

The Xgwm497 marker located in MQTL7 on chromosome $3 \mathrm{~A}$ is relevant to lignin catabolic process and oxygen oxidoreductase activity. Lignification, which is the metabolic process of sealing a plant cell wall by lignin deposition, happens within the course of typical tissue development and is a significant step during root growth. Lignin is one of the final products of phenylpropanoid metabolism and it plays an important role in resistance to biotic and abiotic stresses tolerance (Chen et al., 2013). Some enzymes of this metabolic pathway, such as phenylalanine ammonia-lyase (PAL), tyrosine ammonia-lyase (TAL), and peroxidase (POD), come with the synthesis and polymerization of monolignols that result, in premature lignification (Boerjan et al., 2003; Vanholme et al., 2010). Reduction in root length has been linked to the cell wall lignification induced by allelochemicals. In general, lignification makes the cell wall firm with concomitant increases in PAL and POD activities. In fact, rise of PAL activity is closely connected to decline of root growth and lignin production in maize, cucumber, and soybean vulnerable to the action of phenylpropanoid allelochemicals (Rama Devi and Prasad 1996; Herrig et al., 2002; dos Santos et al., 2004).

Interestingly by most of the identified chromosomal regions resulted were from MQTL analysis involved in the control of a number of different root traits. The meta-analysis reported here will help the selection of targeted root QTLs by marker assisted selection, and provids fundamental information that obtaining QTL-related genes in wheat. These MQTLs associated genomic regions can be further verified by performing fine mapping on large-effect QTLs to narrow down the genomic region responsible for a specific root trait or by developing chromosome segment substitution.

\section{Materials and methods}

\section{QTLs related to root morphology of bread wheat}

Here we analyzed QTLs related to root morphology in wheat under both normal and drought stress conditions resulted from 12 independent experiments published within 20052014 (Table 1). The evaluated root characters included length, surface area, average diameter, volume, number of tips and dry weight. The information on traits, the position of QTLs, chromosome number, confidence interval (CI), phenotypic variance explained by the QTL $\left(\mathrm{R}^{2}\right)$ and LOD values are presented in table 2 . In cases the confidence interval was not available, it was calculated using the following formula with $95 \%$ confidence interval:

$\mathrm{CI}=530 /\left(\mathrm{N} \times \mathrm{R}^{2}\right)$ for $\mathrm{F}_{2}$ lines

$\mathrm{CI}=287 /\left(\mathrm{N} \times \mathrm{R}^{2}\right)$ for $\mathrm{DH}$ lines

$\mathrm{CI}=163 /\left(\mathrm{N} \times \mathrm{R}^{2}\right)$ for RILs

Where $\mathrm{N}$ is the number of lines in the mapping population and $\mathrm{R}^{2}$ is the percentage of phenotypic variation explained by the related QTL.

\section{Analysis of meta-QTL}

The analysis of meta-QTL involved two stages: first the integration of the different genetic maps and then the metaQTL analysis itself. A consensus map contained 1,238 SSR markers spanning 2,569 centiMorgan (cM) with an average of $2.06 \mathrm{cM}$ distance between two adjacent loci (Somers et al., 2004) were used as a reference map for Meta-QTL analysis. The projections of QTLs on the consensus map were performed using BioMercator 4.2 software based on common 
markers among different maps (Arcade et al., 2004) BioMercator is based on the algorithm developed by Goffinet and Gerber (2000) in which a maximum likelihood function is produced to determine (i) the number of MQTLs given a set of input QTLs on a common genetic map, (ii) the consensus position of detected MQTLs based on the variance of input QTLs positions and were estimated as the mean QTLs distribution maximum likelihood, and (iii) a 95\% CI for each MQTLs based on the variance of input QTLs intervals and QTLs is deduced by the algorithm (Arcade et al., 2004): The $95 \%$ confidence interval of the consensus:

$$
C . I .=3.92^{\prime} \sqrt{1 / \stackrel{\circ}{a}_{i=1}^{n}\left(1 / \sigma_{i}^{2}\right)}
$$

where $\sigma \mathrm{i}^{2}$ is the variance of position of the ith QTLs of the distribution. The BioMercator computes the consensus QTLs by models 1, 2, 3, 4 and $n$. Model 1 represents that all the input QTLs are in a single location on the linkage group. Model $\mathrm{n}$ means all the input QTLs are in $\mathrm{n}$ different locations. The model with the lowest Akaike criterion (AIC value) is the most probable model. In each model, a confidence interval is calculated for each detected metaQTLs. The drawing bar chart and chi square test $\left(\chi^{2}\right)$ were performed using Rstudio software. The molecular linkage map was drawn by the Mapchart 2.2 software (Voorrips 2002) (www.wageningenur.nl/en/show/Mapchart.htm).

\section{Exploring functional candidate genes}

The confidence intervals (cM) of identified microsatellite markers linked to MQTL regions on the bread wheat physical map were used to search for the candidate genes in Triticum aestivum on the "Ensembl Plants" website (http://plants.ensembl.org/Triticum_aestivum/Info/Index). Moreover, the sequences were functionally annotated by BLAST analysis, and classified according to the "Uniprot" database (http://www.uniprot.org/).

\section{Conclusion}

Analysis of meta-QTL allowed us to use collected QTLs from different published studies to obtain consensus QTLs across different genetic backgrounds and conditions, and thus provide a better definition on genomic regions involved in the control of root morphological traits and finally chances are there to facilitate the identification of positional candidate genes. Moreover, identified MQTLs can be targeted for future studies on root architecture, breeding and genetic improvement of stress tolerance in bread wheat.

\section{Acknowledgments}

This research was supported by National Institute of Genetic Engineering and Biotechnology of Iran (NIGEB, Grant Number 451M).

\section{References}

Acuña-Galindo MA, Mason RE, Subramanian NK, Hays DB (2014) Meta-analysis of wheat QTL regions associated with adaptation to drought and heat stress. Crop Sci. 55(2): 477492.

Arcade A, Labourdette A, Falque M, Mangin B, Chardon F, Charcosset A, Joets J (2004) Biomercator: integrating genetic maps and QTL towards discovery of candidate genes. Bioinformatics 20: 2324-2326.
Arifuzzaman Md, Sayed MA, Muzammil S, Pillen K, Schumann H, Naz AA, Léon J (2014) Detection and validation of novel QTL for shoot and root traits in barley (Hordeum vulgare L.). Mol Breed. 34(3): 1373-1387.

Austin DF, Lee M, Veldboom LR, Hallauer AR (2000) Genetic mapping in maize with hybrid progeny across testers and generations: grain yield and grain moisture. Crop Sci. 40: 3039.

Boerjan W, Ralph J, Baucher M (2003) Lignin biosynthesis. Ann. Rev. Plant Bio. 54: 519-546.

Burton AL, Johnson J, Foerster J, Hanlon MT, Kaeppler SM, Lynch JP, Brown KM (2015) QTL mapping and phenotypic variation of root anatomical traits in maize (Zea mays L.). Theor Appl Genet. 128(1): 93-106.

Caradus JR (1995) Genetic control of phosphorus uptake and phosphorus status in plants. In: Genetic manipulation of crop plants to enhance integrated nutrient management in cropping system. Patancheru: ICRISAT Asia Centre; pp. 55-67.

Chapagain T, Super L, Riseman A (2014) Root architecture variation in wheat and barley cultivars. Am J Exp Agric. 4(7): 849-856.

Chen HC, Song J, Williams CM, Shuford CM, Liu J, Wang JP, Li Q, Shi R, Gokce E, Ducoste J, Muddiman DC, Sederoff RR, Chiang VL (2013) Monolignol pathway 4-coumaric acid: coenzyme A ligases in Populus trichocarpa: novel specificity, metabolic regulation, and simulation of coenzyme A ligation fluxes. Plant Physiol.161: 1501-1516.

Christopher J, Christopher M, Jennings R, Jones S, Fletcher S, Borrell A, Manschadi AM, Jordan D, Mace E, Hammer G (2013) QTL for root angle and number in a population developed from bread wheat (Triticum aestivum) with contrasting adaptation to water-limited environments. Theor Appl Genet. 126: 1563-574.

Comas LH, Becker SR, Cruz VMV, Byrne PF, Dierig DA (2013) Root traits contributing to plant productivity under drought. Front Plant Sci. 4: 442.

Courtois B, Ahmadi N, Khowaja F, Price AH, Rami J-F, Frouin J, Hamelin C, Ruiz M (2009) Rice root genetic architecture: meta-analysis from a drought QTL database. Rice 2(2): 115 128.

de Dorlodot S, Forster B, Page' L, Price A, Tuberosa R, Draye X (2007) Root system architecture: opportunities and constraints for genetic improvement of crops. Trends Plant Sci. 12: 474481 .

DeYoung BJ, Bickle KL, Schrage KJ, Muskett P, Patel K, Clark SE (2006) The CLAVATA1-related BAM1, BAM2 and BAM3 receptor kinase-like-proteins are required for meristem function in Arabidopsis. Plant J. 45:1-16.

dos Santos WD, Ferrarese MLL, Finger A, Teixeira ACN, Ferrarese-Filho O (2004) Lignification and related enzymes in Glycine max root growth-inhibition by ferulic acid. J Chem Ecol. 30(6): 1203-1212, 2004.

Ehdai B, Waines JG (1997) Chromosomal location of genes influencing plant characters and evapotranspiration efficiency in bread wheat. Euphytica 96: 363-375

Gierth M, Mäser P (2007) Potassium transporters in plants Involvement in $\mathrm{K}^{+}$acquisition, redistribution and homeostasis. FEBS Lett. 581: 2348-2356.

Goffinet B, Gerber S (2000) Quantitative trait loci: a meta analysis. Genetics 155: 463-473.

Hao Z, Li X, Liu X, Xie C, Li M, Zhang D, Zhang S (2010) Meta-analysis of constitutive and adaptive QTL for drought tolerance in maize. Euphytica 174: 165-177.

Herrig V, Ferrarese MLL, Suzuki LS, Rodrigues JD, FerrareseFilho O (2002) Peroxidase and phenylalanine ammonialyaseactivities, phenolic acid contents, and allelochemicals-inhibited root growth of soybean. Biol. Res. 35(1): 59-66.

Horii H, Nemoto K, Miyamoto N, Harada J (2005) Quantitative trait loci for adventitious and lateral roots in rice. Plant Breed. 125: 198-200. 
Khowaja FS, Norton GJ, Courtois B, Price AH (2009) Improved resolution in the position of drought-related QTLs in a single mapping population of rice by meta-analysis. BMC Genomics 10: 276.

Li WT, Liu C, Liu YX, Pu ZE, Dai SF, Wang JR, Lan XJ, Zheng YL, Wei YM (2013) Meta-analysis of QTL associated with tolerance to abiotic stresses in barley. Euphytica, 189: 31-49.

Li YL, Niu SZ, Dong YB, Cui DQ, Wang YZ, Liu YY, Wei MM (2007) Identification of trait-improving quantitative trait loci for grain yield components from a dent corn inbred line in an advanced backcross $\mathrm{BC} 2 \mathrm{~F} 2$ population and comparison with its F2:3 population in popcorn. Theor Appl Genet. 115: 129140.

Li ZK, Yu SB, Lafitte HR, Huang N, Courtois B, Hittalmani S, Vijayakumar CHM, Liu GF, Wang GC, Shashidhar HE, Zhuang JY, Zheng KL, Singh VP, Sidhu JS, Srivantaneeyakul S, Khush GS (2003) QTL $\times$ environment interactions in rice. I. heading date and plant height. Theor Appl Genet. 108: 141153.

Lobet G, Draye X, Périlleux C (2013) An online database for plant image analysis software tools. Plant Methods 9: 38

Maccaferri M, El-Feki W, Nazemi G, Salvi S, Canè MA, Colalongo MC, Stefanelli S, Tuberosa R (2016) Prioritizing quantitative trait loci for root system architecture in tetraploid wheat. J Exp Bot. 67: 1161-1178

MacKey J (1973) The wheat root. In: Sears ER, Sears LMS (eds) Proceedings of the $4^{\text {th }}$ international wheat genetics symposium. University of Missouri Columbia, MO, pp 827-842.

Mao SL, Wei YM Cao W, Lan XJ, Yu M, Chen ZM, Chen GY, Zheng YL (2010) Confirmation of the relationship between plant height and Fusarium head blight resistance in wheat (Triticum aestivum L.) by QTL meta-analysis. Euphytica 174: 343-356.

Marone D, Russo MA, Laidò G, De Vita P, Papa R, Blanco A, Gadaleta A, Rubiales D, Mastrangelo AM (2013) Genetic basis of qualitative and quantitative resistance to powdery mildew in wheat: from consensus regions to candidate genes. BMC Genomics 14: 562.

Monyo JH, Whttington WJ (1970) Genetic analysis of root growth in wheat. J Agric Sci 74: 329-338.

Motzo R, Pruneddu G, Giunta F (2013) The role of stomatal conductance for water and radiation use efficiency of durum wheat and triticale in a Mediterranean environment. Eur $\mathrm{J}$ Agron. 44: 87-97.

Qi ZM, Sun YN, Wu Q, Liu CY, Hu GH, Chen QS (2011) A meta-analysis of seed protein concentration QTL in soybean. Can J Plant Sci. 91: 221-230.

Rama Devi S, Prasad MNV (1996) Ferulic acid mediated changes in oxidative enzymes of maize seedlings: implications in growth Biol Plantarum 38(3): 387-395.

Richard CAI, Hickey LT, Fletcher S, Jennings R, Chenu K, Christopher JT (2015) High-throughput phenotyping of seminal root traits in wheat. Plant Methods 11:13.

Richards RA, Passioura JB (1981) Seminal root morphology and water use in wheat. II. Genetic variation. Crop Sci. 21: 253255.

Rigas S, Desbrosses G, Haralampidis K, Vicente-Agullo F, Feldmann KA, Grabov A, Dolan L, Hatzopoulos P (2001) TRH1 encodes a potassium transporter required for tip growth in Arabidopsis root hairs. Plant Cell 13:139-151.

Sharma S, Villamor JG, Verslues PE (2011) Essential role of tissue-specific proline synthesis and catabolism in growth and redox balance at low water potential. Plant Physiol. 157: 292304

Shiu SH, Bleecker A (2001) Receptor-like kinases from Arabidopsis form a monophyletic gene family related to animal receptor kinases. Proc Natl Acad Sci USA 98:1076310768.

Somers DJ, Isaac P, Edwards K (2004) A high-density microsatellite consensus map for bread wheat (Triticum aestivum L.). Theor Appl Genet. 109: 1105-1114.
Steele KA, Virk DS, Kumar R, Prasad SC, Witcombe JR (2007) Field evaluation of upland rice lines selected for QTLs controlling root traits. Field Crop Res. 101: 180-186.

Steele, K.A. et al. (2006) Marker-assisted selection to introgress rice QTLs controlling root traits into an Indian upland rice variety. Theor Appl Genet. 112: 208-221.

Tavakol E, Sardaro ML, Shariati V, Rossini L, Porceddu E (2014) Isolation, promoter analysis and expression profile of Dreb2 in response to drought stress in wheat ancestors. Gene 549(1): 24-32

Tavakol E, Elbadry N, Tondelli A, Cattivelli L, Rossini L (2016) Genetic dissection of heading date and yield under Mediterranean dry climate in barley (Hordeum vulgare L.). Euphytica 212(2): 343-53.

Tuberosa R, Salvi S, Sanguineti MC, Landi P, Maccaferri M, Conti S (2002a) Mapping QTLs regulating morphophysiological traits and yield: case studies, shortcomings and perspectives in drought-stressed maize. Ann Bot. 89: 941-963.

Tuberosa R, Sanguineti MC, Landi P, Giuliani MM, Salvi S, Conti S (2002b) Identification of QTLs for root characteristics in maize grown in hydroponics and analysis of their overlap with QTLs for grain yield in the field at two water regimes. Plant Mol Biol. 48: 697-712.

Vanholme R, Morree K, Darrah C, Oyarce P, Grabber JH, Ralph J, Boerjan W (2012) Metabolic engineering of novel lignin in biomass crops. New Phytol. 196: 978-1000.

Vicente-Agullo F, Rigas S, Desbrosses G, Dolan L, Hatzopoulos P, Grabov A (2004) Potassium carrier TRH1 is required for auxin transport in Arabidopsis roots. Plant J. 40: 523-535.

Voorrips RE (2002) MapChart: software for the graphical presentation of linkage maps and QTLs. J Hered. 93(1): 77-78.

Wang Y, Xu J, Deng D, Ding H, Bian Y, Yin Z, Wu Y, Zhou B, Zhao Y (2015) A comprehensive meta-analysis of plant morphology, yield, stay-green, and virus disease resistance QTL in maize (Zea mays L.). Planta 243(2): 459-471.

Whalley WR, Dodd IC, Watts CW, Webster CP, Phillips AL, Andralojc J, White RP, Davies WJ, Parry MAJ (2013) Genotypic variation in the ability of wheat roots to penetrate wax layers. Plant Soil 364(1): 171-179.

Wopereis J, Pajuelo E, Dazzo F, Jiang Q, Gresshoff P, de Bruijn F, Stougaard J, Szczyglowski K (2000) Short root mutant of Lotus japonicus with a dramatically altered symbiotic phenotype. Plant J. 23: 97-114.

Wu XL, Hu ZL (2012) Meta-analysis of QTL Mapping Experiments. In: Rifkin SA (ed) Quantitative trait loci (QTL), methods and protocols, Humana Press, New York.

Wu Y, Huang M, Tao X, Guo T, Chen Z, Xiao W (2016) Quantitative trait loci identification and meta analysis for rice panicle-related traits. Mol Genet Genomics 291(5): 1927-1940.

Zhang X, Shabala S, Koutoulis A, Shabala L, Zhou M (2016) Meta-analysis of major QTL for abiotic stress tolerance in barley and implications for barley breeding. Planta 1-13.

Zhang Y, Thomas CL, Xiang J, Long Y, Wang X, Zou J, Luo Z, Ding G, Cai H, Graham NS, Hammond JP, King GJ, White PJ, $\mathrm{Xu}, \mathrm{F}$, Broadley MR, Shi , L, Meng J (2016) QTL metaanalysis of root traits in Brassica napus under contrasting phosphorus supply in two growth systems. Sci. Rep. 6:33113.

Zhao L, Liu HJ, Zhang CX, Wang QY, Li XH (2015) Metaanalysis of constitutive QTLs for disease resistance in maize and its synteny conservation in the rice genome. Genet Mol Res. 14(1): 961-70. 